\title{
DIMENSIONLESS CORRELATION OF LIQUID-PHASE DISPERSION COEFFICIENT IN BUBBLE COLUMNS
}

HaRuo HIKITA* and Hiroshi KIKUKAWA

Department of Chemical Engineering, University of Osaka Prefecture, Sakai, 591

Recently, the authors ${ }^{1)}$ measured the liquid-phase dispersion coefficient in $10 \mathrm{~cm}$ and $19 \mathrm{~cm}$ I.D. bubble columns with single-nozzle gas spargers using air as the gas and using water and aqueous solutions of methanol and cane sugar as liquids. All the experimental data could be correlated well by the following empirical equation:

$$
E_{L}=\left(0.15+0.69 u_{G}^{0.77}\right) D_{T}^{1.25}\left(1 / \mu_{L}\right)^{0.12}
$$

where $E_{L}$ is the liquid-phase dispersion coefficient in $\mathrm{m}^{2} / \mathrm{sec}, u_{G}$ the superficial gas velocity in $\mathrm{m} / \mathrm{sec}, D_{T}$ the column diameter in $\mathrm{m}$, and $\mu_{L}$ the liquid viscosity in cP. This equation, however, is dimensionally inconsistent. In this communication, a new dimensionless correlation is proposed.

\section{Dimensionless Correlation}

Factors which affect the liquid-phase dispersion coefficient $E_{L}$ in bubble columns with single-nozzle gas spargers may be considered to be the column diameter $D_{T}$, the superficial gas velocity $u_{G}$, the density $\rho_{L}$, the viscosity $\mu_{L}$ and the surface tension $\sigma$ of the liquid, and the gravitational acceleration $g$. The effects of the nozzle diameter $d_{0}$ and the aerated liquid height $Z_{F}$ on $E_{L}$ were found in the previous work $^{1)}$ to be negligible. Thus, by dimensional analysis, we obtain:

$$
\frac{E_{L}}{\sqrt{D_{T}^{3} g}}=f\left(\frac{u_{G}}{\sqrt{D_{T} g}}, \frac{\mu_{L}^{4} g}{\rho_{L} \sigma^{3}}, \frac{\rho_{L} D_{T}^{2} g}{\sigma}\right)
$$

Figure 1 presents the experimental data for the waterair system in both columns, $10 \mathrm{~cm}$ and $19 \mathrm{~cm}$ in diameter, as a log-log plot of $E_{L} / \sqrt{D_{T}^{3} g}$ vs. $u_{G} / \sqrt{D_{T} g}$. The data points for two columns of different diameters are seen to fall on a single solid line represented by

$$
\frac{E_{L}}{\sqrt{D_{T}^{3} g}}=0.077+0.39\left(\frac{u_{G}}{\sqrt{D_{T} g}}\right)^{0,72}
$$

This indicates that the effect of the dimensionless group $\rho_{L} D_{T}^{2} g / \sigma$ on $E_{L} / \sqrt{D_{T}^{3} g}$ is unimportant and can be neglected. In Fig. 2, the experimental data obtained with the methanol solution-air system and the cane sugar solution-air system in the $10 \mathrm{~cm}$ column

Received June 5, 1975. are shown as a log-log plot of $E_{L} / \sqrt{D_{T}^{3} g}$ vs. $u_{G} / \sqrt{D_{T} g}$. As can be seen in the figure, the data points for both systems lie about 5 to $30 \%$ below the dotted line representing the data for the water-air system. The

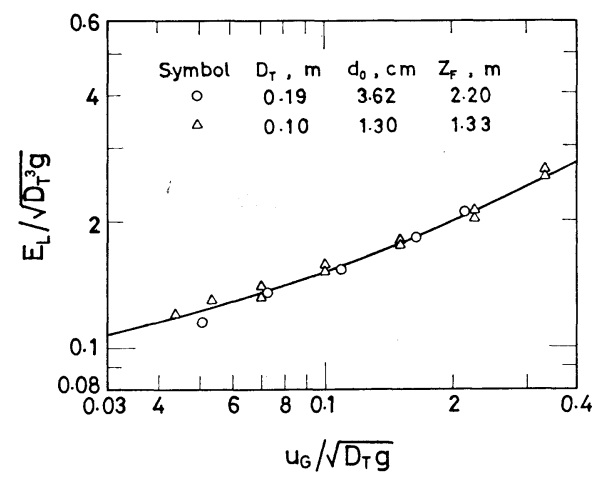

Fig. 1 Liquid-phase dispersion coefficient for water-air system

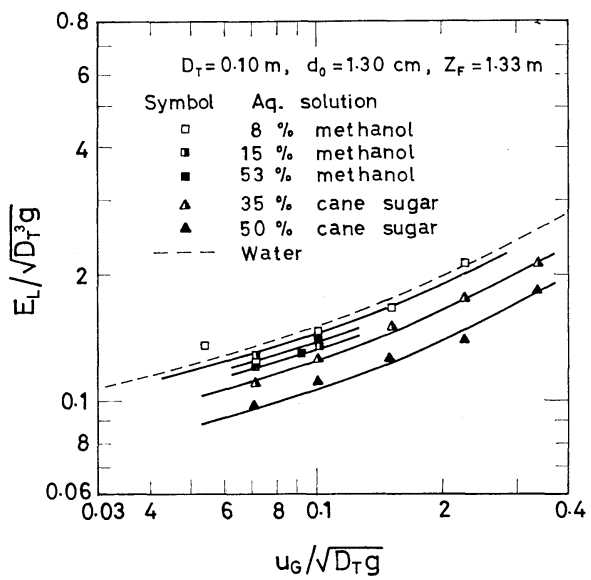

Fig. 2 Liquid-phase dispersion coefficient for methanol solution-air and cane sugar solution-air systems

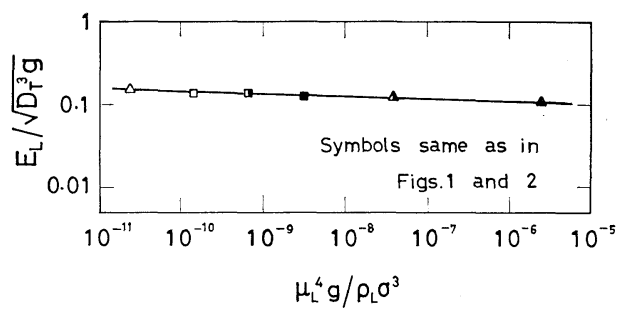

Fig. 3 Effect of physical properties of liquids on liquidphase dispersion coefficient 


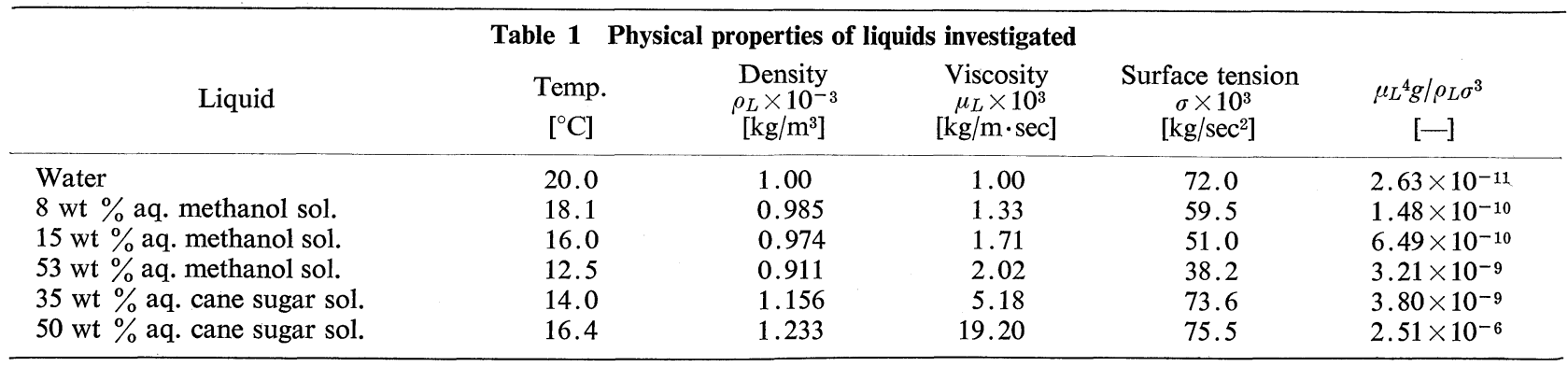

vertical difference among the lines in Fig. 2 may be due to the difference in the value of the dimensionless group $\mu_{L}^{4} g / \rho_{L} \sigma^{3}$. The values of $\mu_{L}^{4} g / \rho_{L} \sigma^{3}$ for the liquids investigated are given in Table 1. In Fig. 3, the values of $E_{L} / \sqrt{D_{T}^{3} g}$ read from Fig. 2 at a constant $u_{G} / \sqrt{D_{T} g}$ value of 0.10 are plotted against $\mu_{L}^{4} g / \rho_{L} \sigma^{3}$ on logarithmic coordinates. Data points fall on a straight line with a slope of -0.03 , indicating that $E_{L} / \sqrt{D_{T}^{3} g}$ should be proportional to $\left(\mu_{L}^{4} g / \rho_{L} \sigma^{3}\right)^{-0.03}$.

From the results described above, the following equation correlating the experimental data was obtained:

$$
\left(\frac{E_{L}}{\sqrt{D_{T}^{3} g}}\right)\left(\frac{\mu_{L}^{4} g}{\rho_{L} \sigma^{3}}\right)=0.037+0.188\left(\frac{u_{G}}{\sqrt{D_{T} g}}\right)^{0.72}
$$

Figure 4 shows the comparison of the observed values of $E_{L}$ presented in Figs. 1 and 2 with the values of $E_{L}$ calculated from Eq. (4). The observed values are in good agreement with the calculated values, with an average deviation of $3.2 \%$.

\section{Nomenclature}

$D_{T} \quad=$ column diameter

$d_{0} \quad=$ diameter of gas inlet nozzle

$E_{L} \quad=$ liquid-phase longitudinal dispersion coefficient

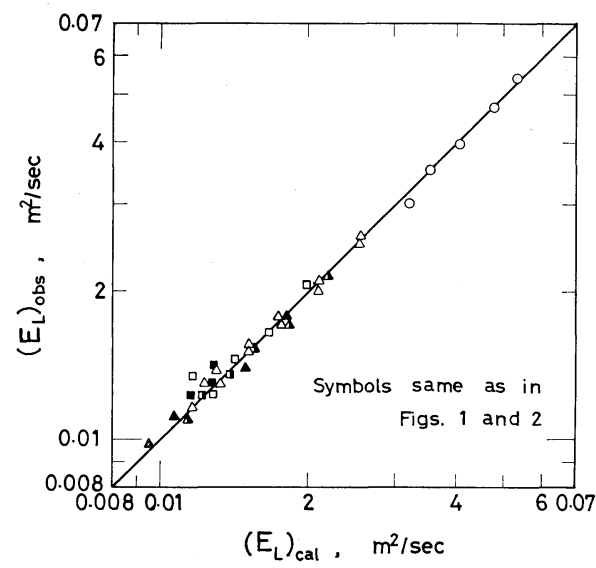

Fig. 4 Comparison of observed and calculated values of liquid-phase dispersion coefficient

$\begin{array}{llr}g & =\text { gravitational acceleration } & {\left[\mathrm{m} / \mathrm{sec}^{2}\right]} \\ u_{G} & =\text { superficial gas velocity } & {\left[\mathrm{m} / \mathrm{sec}^{2}\right.} \\ Z_{F} & =\text { aerated liquid height } & {[\mathrm{m}]}\end{array}$

$\rho_{L} \quad=$ density of liquid $\quad\left[\mathrm{kg} / \mathrm{m}^{3}\right]$

$\mu_{L} \quad=$ viscosity of liquid $\quad[\mathrm{kg} / \mathrm{m} \cdot \mathrm{sec}]$ or $[\mathrm{cP}]$

$\sigma \quad=$ surface tension of liquid $\quad\left[\mathrm{kg} / \mathrm{sec}^{2}\right]$

Literature Cited

[m²/sec] 1) Hikita, H. and H. Kikukawa: Chem. Eng. J., 8, 191 (1974).

\title{
ON THE LOW REYNOLDS NUMBER MASS TRANSFER IN PACKED BEDS
}

\author{
S. N. UPADHYAY*, B. K. D. AGRAWAL AND D. R. SINGH ${ }^{1}$ \\ Department of Chemical Engineering, Institute of Technology, Banaras Hindu University, Varanasi, India
}

Considerable experimental information is available in the literature on the particle-fluid heat and mass transfer in packed beds ${ }^{1,10)}$. For the most part, these efforts are confined to the measurement of transfer rates to gases and liquids flowing at moderate

Received June 19, 1974.

1 Hindustan Zinc Ltd., Udaipur, India and high flow rates. Low rate data, especially for solid-liquid systems, are scanty and are mainly for spherical particles. In the present work new mass transfer rate data are obtained by measuring the rate of dissolution of compressed benzoic acid pellets into water and $60 \%$ aqueous propylene glycol solutions. 